\title{
Caracterização tecnológica de catalisadores automotivos desativados visando o aproveitamento de Pt, Pd e Rh
}

\author{
Renan Teixeira Baia ${ }^{1 *}$ \\ Kleber Bittencourt Oliveira ${ }^{2}$ \\ Emanuel Negrão Macêdo ${ }^{1}$ \\ Jéssica Alves da Silva ${ }^{3}$
}

\section{Resumo}

A utilização de catalisadores automotivos vem aumentando nas últimas décadas. Com as rigorosas legislações de controle de emissões de gases poluentes, o número de catalisadores que são descartados também cresce, gerando enorme volume de resíduos, que podem ser uma fonte para a mineração urbana. O objetivo deste trabalho é caracterizar catalisadores automotivos desativados, em termos da composição mineralógica, estrutural e elementar, quantitativa e qualitativamente. As análises foram realizadas em granulometria por difração à laser, difratômetro de raios X (DRX), microscópio eletrônico de varredura com espectroscopia por dispersão de energia de raios X (MEV/EDS), espectroscopia Raman e espectrometria de emissão óptica em plasma com acoplamento indutivo (ICP-OES). O catalisador automotivo estudado apresentou fissuras e contaminantes como $\mathrm{P}, \mathrm{Ca}, \mathrm{F}, \mathrm{C}$ e S, acentuando o processo de desativação. A maior composição identificada foi de cordierita, correspondendo $40,17 \%$. Os metais catalíticos do grupo platina identificados no catalisador automotivo foram, Pd $(0,92 \%)$, Pt (0,03\%) e Rh $(0,17 \%)$.

Palavras-chave: Catalisadores automotivos; Caracterização; Cordierita; Grupo platina.

\section{Technological characterization of automotive catalysts disabled for Pt, Pd, and $\mathrm{Rh}$ recovery}

\begin{abstract}
s
The use of automotive catalysts has been increasing in recent decades. With the strict regulations governing the emission of polluting gases, the number of catalysts that are discarded also grows, generating enormous volume of waste, which can be a source for urban mining. The objective of this work is to characterize deactivated automotive catalysts in terms of mineralogical, structural and elemental composition, quantitatively and qualitatively. The analyzes were performed in granulometry by laser diffraction, X-ray diffractometer (XRD), scanning electron microscope with X-ray energy dispersion spectroscopy (SEM / EDS), Raman spectroscopy and inductive coupling plasma optical spectrometry (ICP-OES). The studied automotive catalyst presented cracks and contaminants such as $\mathrm{P}, \mathrm{Ca}, \mathrm{F}, \mathrm{C}$ and $\mathrm{S}$, accentuating the deactivation process. The largest composition identified was cordierite, corresponding to $40.17 \%$. The catalysts of the platinum group identified in the automotive catalyst were, $\mathrm{Pd}(0.92 \%), \mathrm{Pt}(0.03 \%)$ and $\mathrm{Rh}(0.17 \%)$.
\end{abstract}

Keywords: Automotive catalysts; Characterization; Cordierite; Platinum group.

\section{Introdução}

O catalisador automotivo é composto principalmente pelo suporte, podendo ser feito de material monolítico cerâmico (cordierita - $2 \mathrm{MgO}_{2} 2 \mathrm{Al}_{2} \mathrm{O}_{3} 5 \mathrm{SiO}_{2}$ ). A sua principal função é proporcionar uma elevada área superficial com uma baixa queda de pressão [1,2]. O suporte monolítico é revestido pelo washcoat, feito de material com área de superfície elevada e resistente à altas temperaturas (geralmente $\mathrm{Al}_{2} \mathrm{O}_{3}$ ), além

\footnotetext{
${ }^{1}$ Programa de Pós-graduação em Engenharia Química, Universidade Federal do Pará-UFPA, Belém, PA, Brasil.

${ }^{2}$ Faculdade de Engenharia Química, Instituto de Tecnologia, Universidade Federal do Pará - UFPA, Belém, PA, Brasil.

${ }^{3}$ Faculdade de Engenharia Química, Universidade do Estado do Amapá - UEAP, Macapá, AP, Brasil.

*Autor correspondente: renanbaiatei@gmail.com
}

2176-1523 (C) 2020. Baia et al. Publicado pela ABM. Este é um artigo publicado em acesso aberto (Open Access) sob a licença Creative Commons Attribution, que permite uso, distribuição e reprodução em qualquer meio, sem restrições desde que o trabalho original seja corretamente citado. 
disso, estão dispersos no washcoat os aditivos de suporte e os metais catalíticos [1,2].

Os aditivos do suporte ajudam a manter a área superficial elevada, são utilizados em temperatura diversas e para separar gases de composições variáveis. Os aditivos também reduzem a sinterização do suporte. Os aditivos podem ser óxidos de lantânio $(\mathrm{La})$, bário $(\mathrm{Ba})$, cério $(\mathrm{Ce})$ ou silício $(\mathrm{Si})[1,3,4]$.

Os metais do grupo platina (Pt, Pd e Rh) estão localizados geralmente na superfície do washcoat [5]. Esses metais proporcionam a redução e oxidação catalítica dos gases poluentes $\left(\mathrm{CO}, \mathrm{HC}\right.$ e $\left.\mathrm{NO}_{\mathrm{x}}\right)$, que são oriundos da queima do combustível pelo motor do automóvel $[4,1]$.

A desativação de catalisadores automotivos geralmente é um fenômeno complexo, principalmente quando envolve catalisadores comerciais, isso devido à presença de muitos componentes que desempenham diversos papéis em todo o sistema catalítico [6-8]. A perda de atividade catalítica e/ou seletividade ao longo do tempo é o resultado de uma série de mudanças químicas e físicas indesejadas [6,9].

A caracterização tecnológica de catalisadores automotivos desativados visa contribuir para o melhor aproveitamento desses resíduos, a partir da identificação e quantificação dos metais de interesse neles contidos [10-12].

O objetivo deste trabalho concentrou-se na caracterização de catalisadores automotivos desativados, em termos de composição mineralógica, estrutural e elementar, quantitativa e qualitativamente. As análises foram realizadas em granulometria por difração à laser, difratômetro de raios $\mathrm{X}(\mathrm{DRX})$, microscópio eletrônico de varredura (MEV), e microscópio eletrônico de varredura com espectroscopia por dispersão de energia de raios $\mathrm{X}$ (MEV/EDS), espectroscopia Raman e espectrometria de emissão óptica em plasma com acoplamento indutivo (ICP-OES).

\section{Materiais e métodos}

\subsection{Material de estudo}

O catalisador automotivo desativado estava em sua carcaça metálica de proteção e com sua manta expansiva interna. A carcaça metálica e a manta expansiva foram retiradas, sobrando assim somente o monolítico cerâmico. Após esse processo, o monolítico foi limpo para retirar o excesso de fuligem oriunda da combustão automotiva. Em seguida, as amostras do monolítico foram moídas em gral ágata e peneiradas para ajustar a granulometria. Após essas etapas, foram realizadas análises de caracterização.

\subsection{Caracterização}

\subsubsection{Análise granulométrica por difração à laser}

Para a análise granulométrica utilizou-se o equipamento Laser Particle Sizer analysette 22 da FritschGmbH, bem como o software MaScontrol, também da FritschGmbH para a aquisição dos dados e geração do gráfico de distribuição granulométrica.

\subsubsection{Difração de Raios X (DRX)}

As análises de DRX foram realizadas pelo método do pó. O difratômetro utilizado foi da marca Bruker, modelo D8 Advance, com detector Lynxeye e com tubo de anodo de $\mathrm{Cu}$ $\left(\mathrm{K}_{\alpha 1}=1,5418\right)$, geometria $(2 \theta / \theta)$, voltagem $40 \mathrm{kv}$ e corrente de $40 \mathrm{~mA}$. Foram usadas as seguintes condições de análise: varredura $5^{\circ}$ a $75^{\circ} 2 \theta$, passo $0,02^{\circ} \mathrm{em} 2 \theta$ e tempo/passo de $1 \mathrm{~s}$.

O objetivo das análises de DRX foi identificar as principais fases cristalográficas que constituem o catalisador automotivo. As amostras do catalisador foram trituradas sem haver separação entre o washcoat e do suporte cerâmico (cordierita).

Para interpretar e tratar os dados foi utilizado o software Match!3 da Crystal Impact. A interpretação dos difratogramas foi feita utilizando-se as fichas da Crystallography Open Database (COD).

\subsubsection{Espectroscopia Raman}

Os espectros Raman foram obtidos em um espectrofotômetro Micro-Raman, marca Jobin Ivon, Modelo T64000, o laser de excitação na linha de 514 nm, potência de operação do laser foi de $17 \mathrm{~mW}$. Os espectros foram gerados por 100 ciclos na região de 100 a $3700 \mathrm{~cm}^{-1}$, com resolução de $0,2 \mathrm{~cm}^{-1}$.

\subsubsection{Microscopia Eletrônica de Varredura (MEV)}

As imagens por microscopia eletrônica de varredura (MEV) foram obtidas no equipamento Hitachi TM3000, acoplado com o Espectrofotômetro de Raios-X de Energia Dispersiva (EDS). Para investigar a morfologia das fases e superfície do catalisador automotivo é utilizado as imagens de microscopia eletrônica de varredura (MEV). O EDS foi responsável pela investigação prévia da composição química do catalisador automotivo.

\subsubsection{Plasma por acoplamento indutivo}

Primeiramente foi realizada uma digestão ácida assistida por radiação micro-ondas do catalisador automotivo, conforme o trabalho de Borisov et al. [13]. Os teores de $\mathrm{Al}, \mathrm{Ba}, \mathrm{Ca}, \mathrm{Fe}, \mathrm{K}, \mathrm{Mg}, \mathrm{Na}, \mathrm{Ni}, \mathrm{P}, \mathrm{Pb}, \mathrm{Pd}, \mathrm{Pt}, \mathrm{Rh}, \mathrm{Si}$, Ti e Zn foram determinados utilizando-se um espectrômetro de emissão óptica em plasma de argônio com acoplamento indutivo da marca Varian, modelo Vista-Pro.

\section{Resultados e discussões}

\subsection{Análise granulométrica}

A distribuição granulométrica do catalisador automotivo está representada pela Figura 1. A distribuição granulométrica indicou que cerca de $90 \%$ das partículas possuem tamanhos menores que $109,7 \mu \mathrm{m} ; 50 \%$ encontram-se abaixo de $43,3 \mu \mathrm{m}$ e $10 \%$ estão abaixo de $7,8 \mu \mathrm{m}$. 


\subsection{Difração de raios-X}

A Figura 2 apresenta o difratograma do catalisador automotivo na região de $5-70^{\circ}$. A análise espectral por DRX em catalisadores automotivos desativados é um processo difícil de ser realizado, pois em sua composição há muitos elementos e contaminantes.

As principais fases encontradas foram: Cordierite ( $\mathrm{Al}_{4} \mathrm{Mg}_{2} \mathrm{Si}_{5} \mathrm{O}_{18}$ - COD ficha n ${ }^{\circ}$ 96-900-5803), óxidos mistos de $\mathrm{Ce}_{0,5} \mathrm{O}_{2} \mathrm{Zr}_{0.5}$ (formula - arquivo COD no 96-210-2843), $\mathrm{LaAlO}_{3}$ (arquivo COD n ${ }^{\circ}$ 96-591-0091), Dióxido de cério $\left(\mathrm{CeO}_{2}\right.$ - arquivo CODn ${ }^{\circ}$ 96-721-7888), Dióxido de zircônio $\left(\mathrm{ZrO}_{2}\right.$ - arquivo COD $\mathrm{n}^{\circ}$ 96-210-1235) e Periclase (MgO - arquivo COD n ${ }^{\circ}$ 96-210-4907).

Os picos característicos da cordierita estão representados no difratograma pelo símbolo (+). A cordierita é o composto que forma a matriz de suporte do catalisador automotivo e representa o material mais abundante do catalisador. Destacam-se as reflexões em $23,940^{\circ} ; 31,725^{\circ}, 45,518^{\circ}$; $56,518^{\circ}$ e $57,430^{\circ}$.

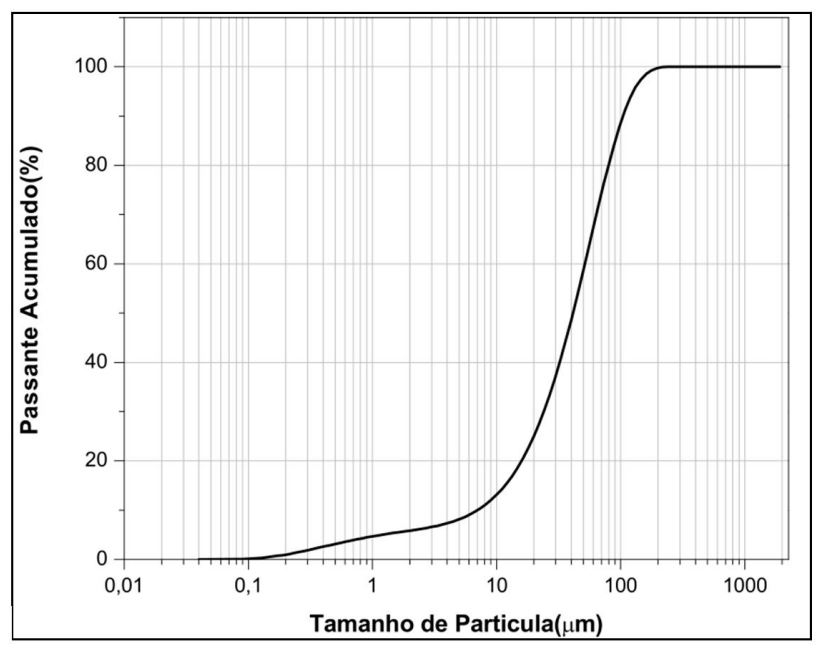

Figura 1. Curva granulométrica do catalisador automotivo.

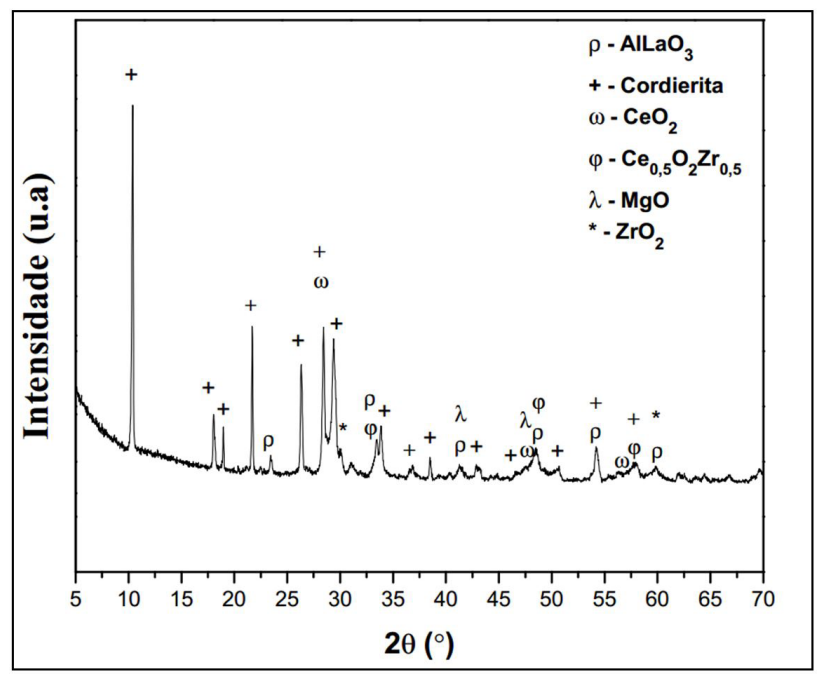

Figura 2. Difratograma do catalisador automotivo.
Foi possível identificar componentes pertencentes ao wascoat do catalisador automotivo. Os principais componentes identificados que fazem parte do wascoat foram: óxidos mistos de $\mathrm{Ce} / \mathrm{Zr}(\varphi), \mathrm{LaAlO}_{3}(\rho), \mathrm{CeO}_{2}(\omega), \mathrm{MgO}(\lambda)$ e $\left.\mathrm{ZrO}_{2}{ }^{*}\right)$. Os picos característicos dos óxidos mistos de Ce/Zr com reflexões em 31,725 e $45,518^{\circ}$; os picos do $\mathrm{CeO}_{2}$ com reflexões em 56,518 $; \mathrm{ZrO}_{2}$ com reflexões em $23,94^{\circ} ; \mathrm{CeO}_{2}$ com reflexões em $56,518^{\circ}$ e $\mathrm{ZrO}_{2}$ com reflexões em $23,94^{\circ}$.

Não foi possível identificar picos característicos da alumina $\left(\mathrm{Al}_{2} \mathrm{O}_{3}\right)$ e suas fases, isso pode ser explicado pela interferência da matriz cerâmica, pois possui picos intensos ou semelhantes ao da alumina e também pela sua baixa cristalinidade [10].

\subsection{Raman}

O espectro Raman do catalisador automotivo pode ser visto na Figura 3. A base literária de espectros Raman de catalisadores automotivos é muito limitada, logo, a análise dos espectros foram baseadas em literaturas dos componentes isolados $[14,15]$.

Os picos que mais se destacam na Figura 3 são de cordierita, representados pelo símbolo $(\alpha)$, esses resultados estão de acordo com os trabalhos de Likhacheva et al. [14] e Majumdar e Mathew [15], que estudaram a cordierita de maneira mais ampla. Os dois picos a 973 e $1185 \mathrm{~cm}^{-1}$ podem ser atribuídos às vibrações internas de alongamento de $\mathrm{SiO}_{4}$ da estrutura de cordierita [16]. O pico $464 \mathrm{~cm}^{-1}$ é característico dos óxidos de cério de catalisadores comerciais de três vias contendo Pd-Rh, como sugere Graham et al. [17].

\subsection{MEV/EDS}

A Figura 4 mostra a micrografia realizada por MEV do perfil da seção transversal do catalisador automotivo, em uma imagem ampliada 40 vezes. Pode-se observar na Figura 4 os canais regulares (estrutura semelhante a "favos de mel") do suporte monolítico, que funcionam como base de sustentação para o catalisador automotivo, e ao redor desses canais observa-se uma distribuição uniforme do washcoat.

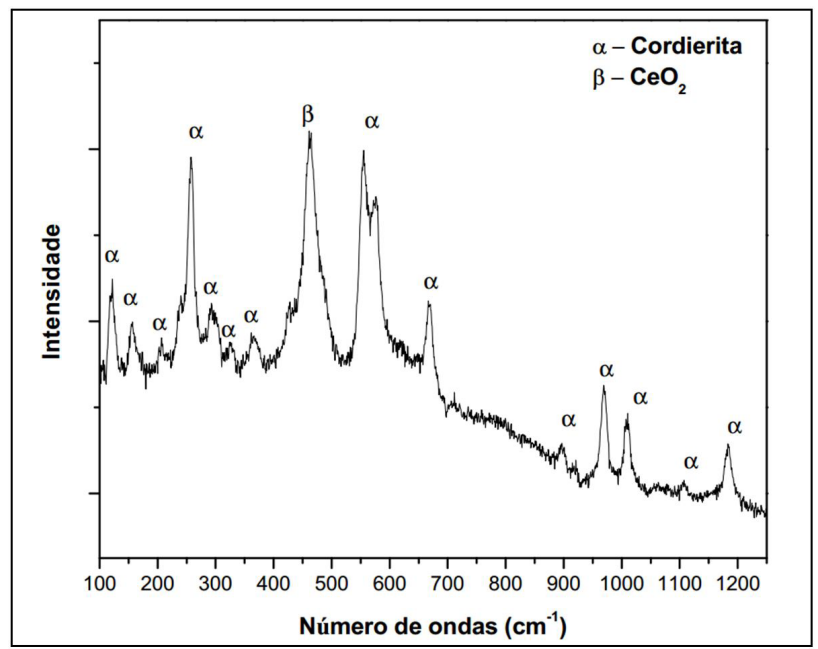

Figura 3. Espectros Raman do catalisador automotivo. 
A Figura 5 é uma ampliação da Figura 4 na área $\mathrm{A}_{0}$, aumentada 200 vezes. Na Figura 5 observa-se de forma mais nítida e homogênea o suporte cerâmico (cordierita), que é o principal componente do catalisador automotivo, está localizado no centro da imagem e em forma de cruz. Envolto ao suporte cerâmico é observado o washcoat, contendo seus óxidos mistos, que podem ser identificados pela divisão de coloração na imagem. Outro fato importante destacado no washcoat que pode ser observado na Figura 5 é a presença de fissuras acentuando os sinais de deterioração do catalisador automotivo.

Os pontos 1, 2, 3 e 4 marcados na Figura 5 foram analisados com o detector EDS e seus respectivos espectros e composição elementar, em termos de porcentagem de massa, são apresentados na figura 6.

Foram encontrados no ponto 1 (espectro 1) os elementos: $\mathrm{Al}$ (alumínio), $\mathrm{Mg}$ (magnésio), $\mathrm{Si}$ (sílica) e $\mathrm{O}$ (oxigênio), que são os elementos básicos que compõem a cordierita $\left(\mathrm{Al}_{4} \mathrm{Mg}_{2} \mathrm{Si}_{5} \mathrm{O}_{18}\right)$, que, por sua vez, é o elemento básico do suporte do catalisador automotivo, também foram encontrados os elementos $\mathrm{Ca}$ (cálcio), Zn (zinco) e P (fósforo),

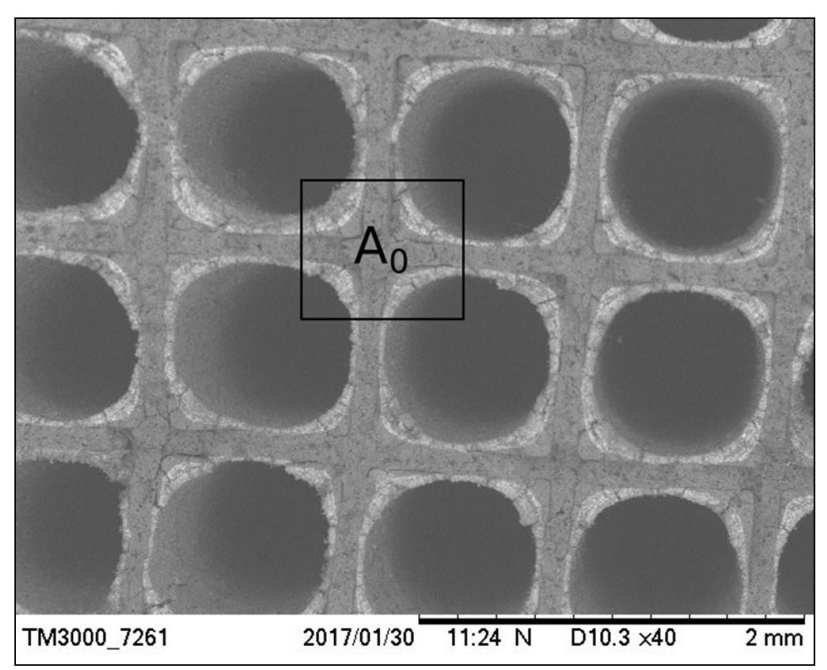

Figura 4. Micrografia do catalisador automotivo, ampliada 40 vezes.

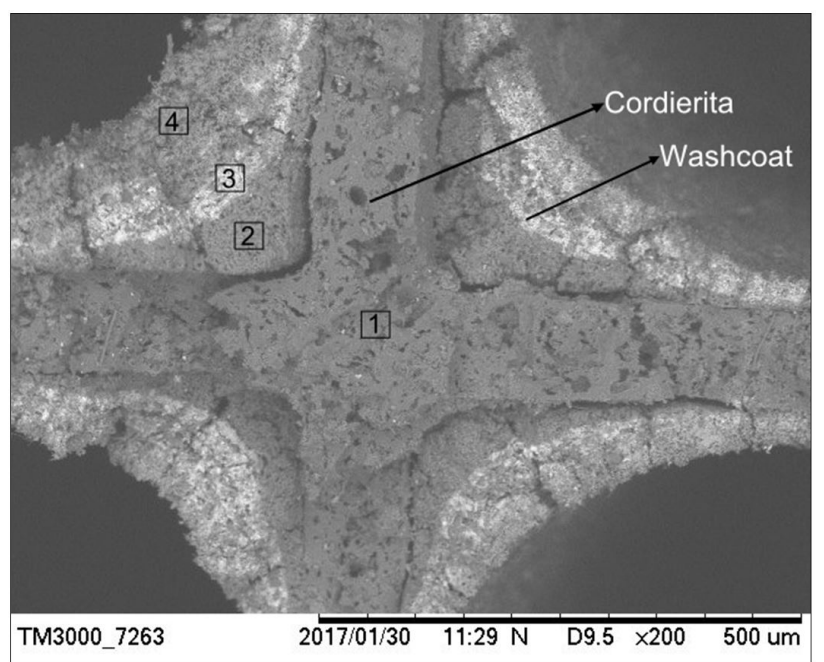

Figura 5. Micrografia do catalisador automotivo, ampliada 200 vezes. que podem ser contaminantes oriundos da queima de aditivos adicionados em combustível e/ou de óleos lubrificantes que contém esses elementos em sua composição, como analisado nos trabalhos da literatura $[18,19]$.

O ponto 2 (espectro 2) corresponde a uma região mais interna do washcoat, os elementos identificados nessa região foram: $\mathrm{O}, \mathrm{Al}, \mathrm{Mg}, \mathrm{Si}, \mathrm{Ca}$. Nota-se uma alta concentração de Alumínio (Al), sugerindo que nesse ponto se encontra uma camada de $\mathrm{Al}_{2} \mathrm{O}_{3}$, que é o componente mais abundante do washcoat.

O ponto 3 corresponde à região catalítica do washcoat. Foi identificada a presença de $\mathrm{Al}, \mathrm{Ce}, \mathrm{Mg}$, Si e Zr pelo espectro 3 , sugerindo a presença de óxidos mistos típicos de washcoat, como $\mathrm{Al}_{2} \mathrm{O}_{3}, \mathrm{CeO}_{2}, \mathrm{MgO}, \mathrm{SiO}_{2}$ e ZrO . Foram identificados Pde $\mathrm{Rh}$, componentes catalíticos do washcoat. Não foram encontrados vestígios de platina (Pt). O ponto 4 (espectro 4) corresponde a uma região fora do washcoat que contem principalmente fuligem e outros contaminantes (C, Si, P, Ca).

\subsection{Análise química}

A análise química obtida do catalisador automotivo foi realizada por ICP-OES, a partir de uma digestão ácida assistida por radiação micro-ondas, com temperatura e pressão controladas. A Tabela 1, mostra os elementos encontrados e quantificados em porcentagem da massa total (\%).

As maiores composições encontradas na Tabela 1, foram de $\mathrm{Al}, \mathrm{Si}$ e $\mathrm{Mg}$, elementos correspondentes à cordierita, que totaliza $40,17 \%$ da massa total do catalisador analisado. Os metais do grupo platina ( $\mathrm{Pd}, \mathrm{Pt}$ e $\mathrm{Rh}$ ) foram identificados em proporções pequenas, correspondendo a $\mathrm{Pd}(0,92 \%), \mathrm{Pt}$ $(0,03 \%)$ e $\mathrm{Rh}(0,17 \%)$ da massa total do catalisador.

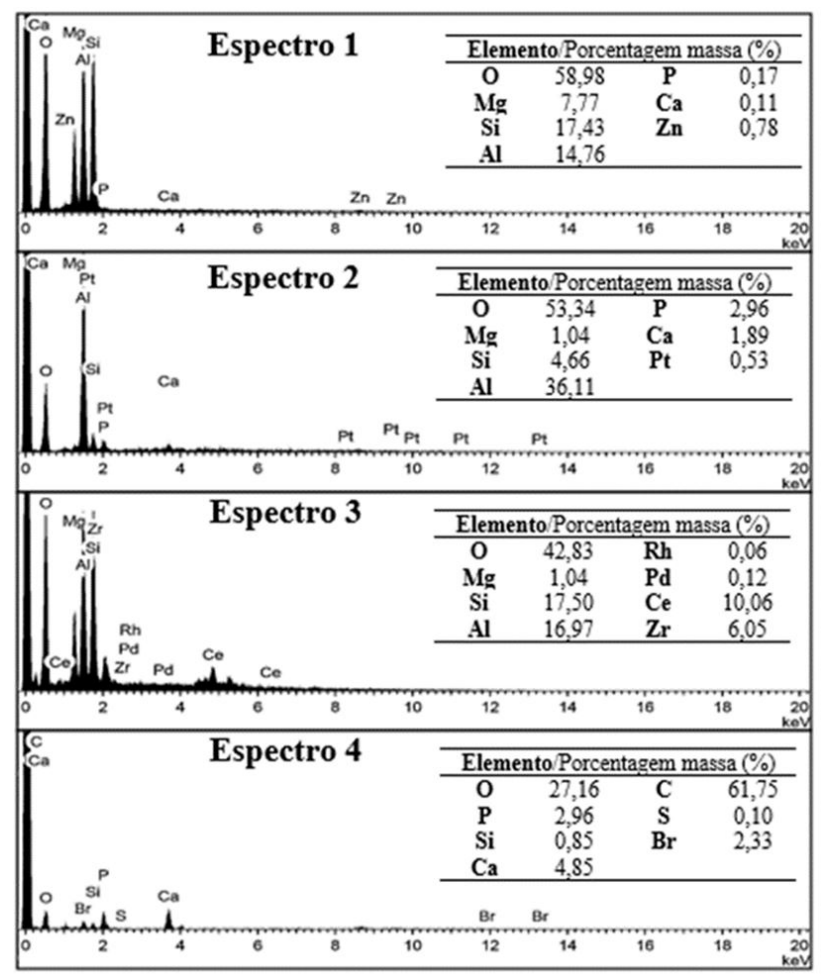

Figura 6. Espectros de EDS do catalisador automotivo. 
Tabela 1. Composição química do catalisador automotivo

\begin{tabular}{ccc}
\hline Elemento & $\begin{array}{c}\text { Porcentagem de massa } \\
(\%)\end{array}$ & $\begin{array}{c}\text { Limite de deteç̧ão } \\
(\mathbf{p p m})\end{array}$ \\
\hline $\mathrm{Al}$ & $19,51 \%$ & 0,040 \\
$\mathrm{Ba}$ & $1,90 \%$ & 0,010 \\
$\mathrm{Ca}$ & $1,40 \%$ & 0,010 \\
$\mathrm{Fe}$ & $1,39 \%$ & 0,010 \\
$\mathrm{~K}$ & $0,83 \%$ & 0,010 \\
$\mathrm{Mg}$ & $7,89 \%$ & 0,030 \\
$\mathrm{Na}$ & $2,48 \%$ & 0,030 \\
$\mathrm{Ni}$ & $0,87 \%$ & 0,015 \\
$\mathrm{P}$ & $0,95 \%$ & 0,010 \\
$\mathrm{~Pb}$ & $0,02 \%$ & 0,040 \\
$\mathrm{Pd}$ & $0,92 \%$ & 0,100 \\
$\mathrm{Pt}$ & $0,03 \%$ & 0,100 \\
$\mathrm{Rh}$ & $0,17 \%$ & 0,100 \\
$\mathrm{Si}$ & $12,77 \%$ & 0,100 \\
$\mathrm{Ti}$ & $0,21 \%$ & 0,100 \\
$\mathrm{Zn}$ & $0,16 \%$ & 0,005 \\
\hline
\end{tabular}

\section{Conclusão}

A caracterização dos catalisadores automotivos desativados descartados deste estudo mostrou que possuem várias substâncias de interesse comercial, como paládio $(\mathrm{Pd})$, platina $(\mathrm{Pt})$ e ródio $(\mathrm{Rh})$, que são utilizados principalmente na fabricação de novos catalisadores automotivos. Esses elementos ainda estão presentes em quantidades suficientes para justificar serem alvos de processo de extração e reciclagem das mesmas.

Tal conduta, além de ter potencial para dar retornos econômicos expressivos, pode, também, contribuir com a preservação do meio ambiente por ser uma fonte alternativa que poupa novas extrações da natureza e diminui a quantidade exorbitante de lixo automotivo gerado e estocado em nosso país, que muitas vezes é descartado de forma inadequada, gerando riscos incalculáveis ao meio ambiente e aos seres vivos em geral.

\section{Agradecimentos}

Os autores agradecem a colaboração técnicocientífica da Universidade Federal do Pará (UFPA) e financeira do Conselho Nacional de Desenvolvimento Científico e Tecnológico (CNPQ).

\section{Referências}

1 Heck RM, Farrauto RJ. Automobile exhaust catalysts. Applied Catalysis A, General. 2001;221(1):443-457. http:// dx.doi.org/10.1016/S0926-860X(01)00818-3.

2 Russell A, Epling WS. Diesel oxidation catalysts. Catalysis Reviews. 2011;53(4):337-423. http://dx.doi.org/10.1080/ 01614940.2011 .596429$.

3 Kato A, Yamashita H, Kawagoshi H, Matsuda S. Preparation of Larnthanum $\beta$-alumina with high surface area by coprecipitation. Journal of the American Ceramic Society. 1987;70(7):7. http://dx.doi.org/10.1111/j.1151-2916.1987. tb05694.x.

4 Wallington TJ, Kaiser EW, Farrell JT. Automotive fuels and internal combustion engines: a chemical perspective. Chemical Society Reviews. 2006;35(4):335-347. http://dx.doi.org/10.1039/b410469m.

5 Moldovan M, Milagros Gómez M, Antonia Palacios M. Determination of platinum, rhodium and palladium in car exhaust fumes. Journal of Analytical Atomic Spectrometry. 1999;14(8):1163-1169.

6 Koltsakis GC, Stamatelos AM. Catalytic automotive exhaust aftertreatment. Progress in Energy and Combustion Science. 1997;23(1):1-39.

7 Lassi U. Deactivation correlations of $\mathrm{Pd} / \mathrm{Rh}$ three-way catalysts designed for Euro IV emission limits: effect of ageing atmosphere, temperature and time [dissertation]. Oulu: University of Oulu; 2003.

8 Fernandes DM, Scofield CF, Alcover A No, Cardoso MJB, Zotin FMZ. Thermal deactivation of Pt/Rh commercial automotive catalysts. Chemical Engineering Journal. 2010;160(1):85-92. http://dx.doi.org/10.1016/j. cej.2010.03.013.

9 Carol LA, Newman NE, Mann GS. High temperature deactivation of three-way catalyst. Journal of Fuels and Lubricants. 1989;98(4):731-744.

10 Zotin FMZ, Gomes ODFM, Oliveira CH, Alcover A No, Cardoso MJB. Automotive catalyst deactivation: case studies. Catalysis Today. 2005;107:157-167. http://dx.doi.org/10.1016/j.cattod.2005.07.111.

11 Batista CH, Dutra AJB. Caracterização de catalisadores automotivos novos e usados visando à reciclagem dos metais. Revista Matéria. 2013;18(4):1451-1458. 
12 Fernandes DM, Scofield CF, Alcover A No, Cardoso MJB, Zotin FMZ, Batista CH, et al. The influence of temperature on the deactivation of commercial $\mathrm{Pd} / \mathrm{Rh}$ automotive catalysts. Environment and Progress. 2009;87(5):315-322.

13 Borisov OV, Coleman DM, Oudsema KA, Carter RO 3rd. Determination of platinum, palladium, rhodium and titanium in automotive catalytic converters using inductively coupled plasma mass spectrometry with liquid nebulization. Journal of Analytical Atomic Spectrometry. 1997;12(2):239-246.

14 Likhacheva AY, Goryainov SV, Krylov AS, Bul'bak TA, Prasad PS. Raman spectroscopy of natural cordierite at high water pressure up to GPa. Journal of Raman Spectroscopy. 2012;43(4):559-563.

15 Majumdar AS, Mathew G. Raman-infrared (IR) spectroscopy study of natural cordierites from Kalahandi, Odisha. Journal of the Geological Society of India. 2015;86(1):80-92.

16 You J, Jiang G, Hou H, Chen H, Wu Y, Xu K. Quantum chemistry study on superstructure and Raman spectra of binary sodium silicates. Journal of Raman Spectroscopy. 2005;36(3):237-249.

17 Graham G, Weber W, McBride J, Peters C. Raman investigation of simple and complex oxides of platinum. Journal of Raman Spectroscopy : JRS. 1991;22(1):1-9.

18 McArthur DP. Deposition and distribution of lead, phosphorus, calcium, zinc, and sulfur poisons on automobile exhaust NOx catalysts. Catalysts for the Control of Automotive Pollutants. 2009;143:85-102.

19 Angelidis TN, Sklavounos SA. A SEM-EDS study of new used automotive catalysts. Applied Catalysis A, General. 1995;133(1):121.

Recebido em: 13 Fev. 2019

Aceito em: 15 Fev. 2020 